\title{
Envejecer es crecer. Reaprendiendo día a día
}

\author{
Claudia Esteban Fulleda \\ al262942@uji.es \\ AIDA MARTínez PÉREZ \\ al262759@uji.es \\ MARÍA PILAR MolÉs JULIO \\ mjulio@uji.es
}

\section{Resumen}

Introducción: Las patologías de Parkinson y la demencia son de gran importancia en las personas de edad avanzada, de ahí nuestro interés en ellas. Este estudio trata de un varón de 80 años que padece ambas patologías. Nuestro objetivo es crear un plan de cuidados enfermeros para aumentar su independencia, además de su calidad de vida. Método: El plan de cuidados se basó principalmente en ejercicios de rehabilitación, prevención del riesgo de caídas, estimulación multisensorial y en talleres de aprendizaje. Resultados: Los resultados han sido satisfactorios para los objetivos establecidos, ya que observamos un aumento de la independencia del paciente y una mejora en su calidad de vida. Conclusiones: Se puede concluir que si se individualizan los cuidados se obtiene una mejora en la calidad de vida y un aumento de su autonomía.

Palabras clave: demencia, Parkinson, cuidados de enfermería, dependencia.

\begin{abstract}
Introduction: The pathology of Parkinson and dementia are of great importance in the elderly, hence our interest in them. This study is about a man of 80 who suffers from both diseases. Our goal is to create a plan of nursing care to prevent the progression of the disease and increase their independence, in addition to his quality life. Method: The care plan was based mainly on rehabilitation exercises, preventing the risk of falls, multisensory stimulation and learning workshops. Results: The results have been satisfactory according to the objectives set, as we observed an increased patient independence and improved quality of life. Conclusions: We may conclude that if cares are individualized, an improvement in the quality of life and a higher autonomy can be obtained.
\end{abstract}

Keywords: dementia, Parkinson, nursing, care. 


\section{Introducción}

La enfermedad de Parkinson pertenece a un grupo de enfermedades llamadas trastornos del movimiento. Los cuatro síntomas principales de esta enfermedad son: temblores (en manos, brazos, piernas, mandíbula y cabeza), rigidez o agarrotamiento de las extremidades y el tronco, bradicinesia (lentitud en los movimientos) e inestabilidad postural. Por lo general, estos síntomas se manifiestan gradualmente y se agravan con el paso del tiempo. A medida que se vuelven más pronunciados, los pacientes pueden tener dificultad para caminar, hablar o completar otras tareas sencillas (De la Casa Fages, 2014).

Por su parte, la demencia es un síndrome, generalmente de naturaleza crónica o progresiva, caracterizado por el deterioro de la función cognitiva, es decir, la capacidad para procesar el pensamiento más allá de lo considerado envejecimiento normal. Sus signos y síntomas asociados no son bien conocidos, lo que dificulta una detección y un diagnóstico precoz, complicando así los cuidados y tratamientos aplicados a dichos pacientes (Anderson, GonzálezCosío, y Resnikoff, 2014).

La demencia afecta sobre todo a las personas de edad avanzada. El $20 \%$ de todas las personas con más de 80 años padece demencia. Debido al envejecimiento creciente de la población, la demencia se está convirtiendo en un problema de gran magnitud (OMS, 2015).

Según el Instituto Nacional de Trastornos Neurológicos y Accidentes Cerebrovasculares el Parkinson afecta alrededor del 50 \% más de hombres que mujeres. Un factor de riesgo para la enfermedad es la edad. La edad promedio es de 60 años. Sin embargo, entre el $5 \%$ y el $10 \%$ de las personas con enfermedad de Parkinson tienen una enfermedad de «inicio temprano» que comienza antes de los 50 años de edad. Las personas con uno o más familiares con la enfermedad tienen un aumento de riesgo de contraer ellos mismos la enfermedad, el riesgo es de $2 \%$ a $5 \%$. En casos muy raros, los síntomas parkinsonianos pueden aparecer en las personas antes de los 20 años (parkinsonismo juvenil), a menudo es hereditario. Y la incidencia global mundial de la demencia se estima alrededor de 7,5/1000 personas-año. La aparición de nuevos casos se mantiene más o menos estable hasta los 65-70 años. La incidencia anual de demencia en España es de 10-15 casos por 1000 personas-año en la población mayor de 65 años (De la Casa Fages, 2014).

La enfermedad de Parkinson y la demencia afectan tanto al individuo como al grupo familiar. Implican trastornos físicos, psicológicos, emocionales, económicos y sociales que deben ser asumidos por el usuario y su familia. Requieren la constante adaptación familiar y conllevan mayor riesgo de aislamiento social, maltrato y desvinculación. Estas enfermedades comprometen todas las áreas del funcionamiento y requieren una evaluación multidimensional. El objetivo del tratamiento es controlar los síntomas y mantener la mejor funcionalidad posible y conservar la calidad de vida de pacientes y sus cuidadores (Janssen, sf; Romero y cols., 2014). La utilidad de terapia ocupacional y educación a cuidadores para mejorar la calidad de vida de los pacientes ha sido previamente aplicada y evaluada de forma multidimensional (Rosson y cols., 2013).

\section{Objetivos}

\section{Objetivo general}

Analizar la evolución de las patologías en base al plan de cuidados enfermero que le proporcionamos. 


\section{Objetivo secundario}

Proporcionar una mejoría de su independencia.

\section{Método}

La elección del caso queda justificada por la prevalencia de demencia y Parkinson en nuestra sociedad, ya que afecta a un número elevado de personas de avanzada edad.

El estudio se centra en un varón institucionalizado en la residencia de la tercera edad de Borriana en situación de dependencia, que padece varias patologías, entre las cuales se identifican la demencia y la enfermedad de Parkinson, además padece hipotensión arterial e insomnio nocturno. Nuestro paciente es un varón (E. M. S.) de 80 años de edad, con un peso de $92 \mathrm{~kg}$ y una talla de $187 \mathrm{~cm}$, alérgico a la penicilina, con reacciones adversas a neurolépticos y diazepam en tomas continuas. El paciente ingresa el 18 de octubre de 2014 a petición de la familia, ya que en los últimos 2 meses ha sufrido un deterioro físico y mental muy acelerado.

Se trata de un estudio epidemiológico, descriptivo y prospectivo con medidas repetidas, de un mes de duración. Para realizar este estudio hemos utilizado diversas escalas validadas incluidas en la valoración geriátrica integral, como el test de Pfeiffer para la valoración del deterioro cognitivo, la escala Morse para la evaluación del riesgo de caídas, test de Barthel, que evalúa el grado de dependencia, la escala EVA para la valoración subjetiva del dolor, la escala de Norton, que evalúa el riesgo de UPP, y el test de Hermes, para evaluar la adherencia al tratamiento; así como entrevistas con el paciente y sus familiares, a fin de establecer un plan de cuidados enfermeros adaptados a la situación actual del paciente.

El plan de cuidados desarrollado y que fue proporcionado al paciente consiste en:

1. Ejercicios de rehabilitación para una mejora de la marcha y el equilibrio (ejercicios de Frenkel y talón-planta-punta).

2. Ejercicios para la mejora de la motricidad fina y motricidad gruesa (encajar piezas de puzles, encestar la pelota en un aro, etc.).

3. Prevención del riesgo de caídas buscando el correcto centro de gravedad.

4. Estimulación del paciente en la sala multisensorial con actividades para la mejora de la utilización de objetos cotidianos (abrir y cerrar pomos y grifos, marcar números de teléfono, etc.).

5. Animar a la participación en talleres, como el de francés, que mejoró la relación del paciente con otros usuarios.

6. Controles periódicos de la tensión arterial debido a su hipotensión, durante las dos primeras semanas de manera diaria, y cuando se estabilizaron las cifras, controles semanales.

7. Curas por herpes Zóster en cuero cabelludo con suero fisiológico y povidona yodada cada dos días durante dos semanas.

8. Administración de medicación para el dolor.

\section{Resultados}

Tras la aplicación del plan de cuidados se evidencia una mejora del estado cognitivo, ya que se consigue tener una conversación coherente con el paciente; con respecto al Parkinson, mejoró la marcha, la motricidad fina y la motricidad gruesa, el equilibrio y la coordinación, ya 
que en el momento del ingreso no conseguía andar más de $3 \mathrm{~m}$ de forma continua y sin las paralelas y al alta andaba unos $10 \mathrm{~m}$ con ayuda de andador. Observamos una mejoría en su hipotensión arterial, consiguiendo espaciar los controles de tensión arterial. Por otro lado, disminuyó el insomnio provocado por un herpes Zóster en el cuero cabelludo, gracias a las curas periódicas y la administración de medicación calmante cuando precisaba.

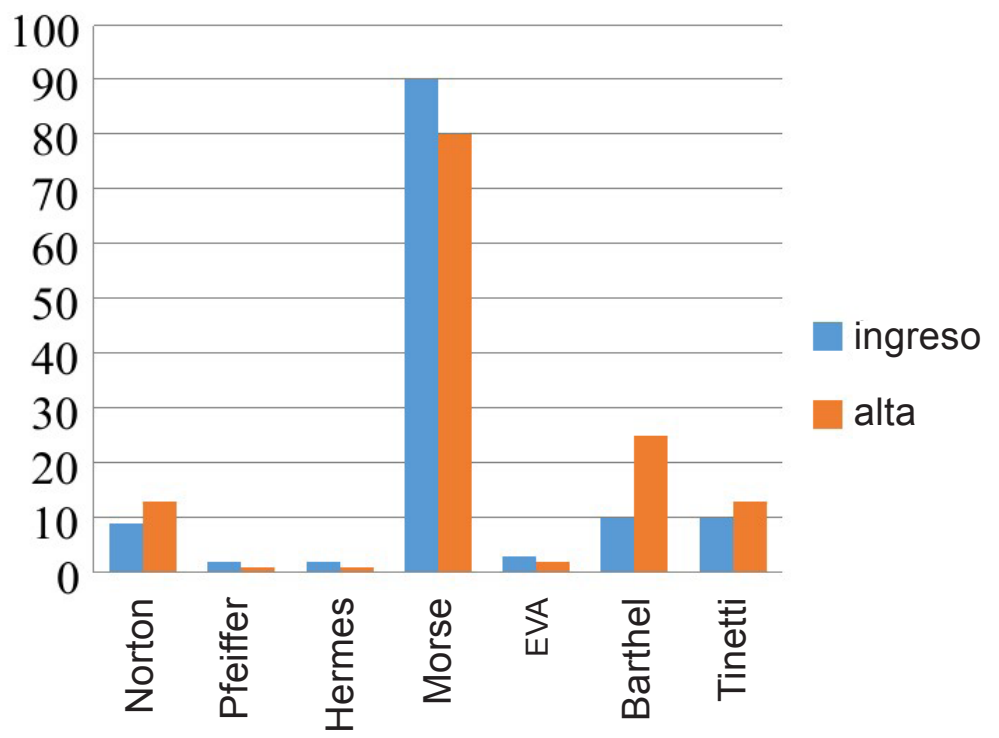

Figura 1. Resultados obtenidos en las escalas incluidas en la valoración geriátrica integral al ingreso y al alta

Como podemos observar (figura 1), se obtuvo una mejora en los resultados al alta con respecto al ingreso, ya que en la escala Norton (1987) se obtuvo un incremento al alta de un $25 \%$ respecto al ingreso gracias a la mejora de la actividad del paciente. En la escala Pfeiffer (1975), se observó una mejora al haber un $25 \%$ menos de errores a las preguntas expuestas, viendo que su adaptación a la residencia era buena. En cuanto a la escala Hermes, mejoró un 33,3 \% al alta, debido a que se toma la medicación bajo la supervisión de enfermería. La puntuación de la escala Morse (Morse, Morse, y Tylko, 1989) es un 10 \% decreciente, aunque aún hay riesgo de caídas, es menor que a su ingreso, al igual que la escala Tinetti (1986), que muestra una mejora en el equilibrio y la marcha debido a sus ejercicios fisioterapéuticos. La escala EVA (Scott y Huskisson, 1979) muestra una disminución del dolor debido al tratamiento para la cura del herpes zóster del cuero cabelludo. Y por último, en la escala Barthel (Mahoney y Barthel, 1965) vemos que ha aumentado un $15 \%$ su puntuación, lo que indica que tiene menor dependencia que a su ingreso, aunque cabe destacar que sigue siendo dependiente total. 


\section{Discusión}

Según el estudio de Salgado y Espinosa (2012) el tratamiento de referencia para la enfermedad del Parkinson debería implicar un equipo multidisciplinar con atención adaptada a sus necesidades específicas y a las de su familia y cuidadores.

El estudio de Rosson y cols. (2013) recomienda actividades promocionales, preventivas, educacionales y la colaboración de un terapeuta ocupacional, Atención Primaria de Salud (APS) puede aportar en mejorar la vitalidad, disminuir la sobrecarga y ayudar a mejorar la calidad de vida en el componente de salud mental del cuidador de pacientes con demencia o Parkinson. Esto impacta en la vida familiar y en la demanda sobre el sistema de salud. Estas intervenciones son de bajo costo y pueden ser llevadas a cabo por cualquier equipo de APS.

Comparando los estudios de Salgado y Espinosa (2012) y Rosson y cols. (2013) con los resultados obtenidos en nuestro plan de cuidados, coincidimos en realizar un abordaje desde el punto de vista de rehabilitación mediante reeducación de la marcha, ejercicio físico, trabajo con texturas, ejercicios aeróbicos, ejercicios de alternancia y coordinación, fortalecimiento muscular y movilizaciones. Además de aplicar cuidados enfermeros específicos como adaptación de la nutrición, medidas preventivas de desarrollo de úlceras por presión, animar al familiar a dar paseos con el paciente, y utilización de escalas de valoración enfermera para ver sus progresos.

El estado cognitivo mejoró de manera positiva, ya que se identificaron y abordaron aspectos relacionados en la calidad de vida del paciente. Basándonos en el estudio de Carmona y Murgui (2015), las personas mayores pueden alcanzar una edad muy avanzada sin presentar alteración cognitiva alguna, manteniendo una actividad mental significativa y de calidad. Sin embargo, algunas personas mayores muestran manifestaciones aisladas, como amnesia y desorientación, entre otras, síntomas que se asocian a lo que se ha denominado deterioro cognitivo leve y que presenta, al menos, dos lecturas.

Algunos especialistas, en el estudio de Carmona y Murgui (2015) opinan que el deterioro cognitivo leve forma parte de manifestaciones pre-Alzheimer, frente a otros que consideran esas manifestaciones como un deterioro mental asociado al envejecimiento.

\section{Conclusión}

Atendiendo a los resultados obtenidos en el estudio actual, se considera que el plan de cuidados desarrollado en un paciente con diagnósticos de demencia y enfermedad de Parkinson resulta adecuado, ya que comprobamos una mejora de su independencia.

El abordaje del paciente con pluripatología requiere una intervención de enfermería centrada en la implantación de planes de cuidados individualizados que permitan potenciar la autonomía del paciente, lo cual influirá en una mejora de la calidad de vida unida a una mejor percepción de su estado de salud, y con resultados favorables en su salud general.

\section{Referencias bibliográficas}

Anderson, R., González-Cosío, M. y Resnikoff, D. (sf). Manual para el cuidador de pacientes con demencia. Recomendaciones para un cuidado de calidad. Recuperado el 18 de noviembre de 2014, de http://www.copib.es/pdf/Vocalies/envelliment/manual\%20cuidadores.pdf. 
Carmona, José V. y Murgui, S. (2015). Cuidados de enfermería en el paciente con enfermedad de Alzheimer: XII Curso de Atención Farmacéutica: Deterioro cognitivo y demencias. Módulo 3, tema 15. El farmacéutico: profesión y cultura, 515, 46-50.

De la Casa Fages, B. (sf). Guía Informativa de la Enfermedad de Parkinson. Federación Española de Parkinson (FEP). Recuperado el 20 de noviembre de 2014, de http://www.fedesparkinson.org/upload/aaff_guia_parkinson.pdf.

Janssen (sf). Demencia. Recuperado el 6 de mayo de 2015, de http://www.janssen.es/salud/ neurociencia/demencia.

Mahoney, FI. y Barthel, D. W. (1965). Functional evaluation: the Barthel Index. Maryland Medical Journal, 14, 61-65.

Morse, J. M., Morse, R. M. y Tylko, S. J. (1989). Development of a scale to identify the fallprone patient. Canadian Journal on Aging, 8, 366-377.

Norton, D. (1987). Norton revised risk scores. Nursing Times, 83, 6.

OMs (2015) Demencia. Recuperado el 2 de mayo de 2014, de http://www.who.int/mediacentre/ factsheets/fs362/es/.

Pfeiffer, E. (1975). A short portable mental status questionnaire for the assessment of organic brain deficit in elderly patients. Journal of American Geriatric Society, 23, 433-441.

Romero, L., Esquivel, A., Mesa, M., Mohedas, A., y Recarte, M. (2014). Fisioterapia en las enfermedades neurológicas en el anciano. Reduca, 6, 195-201.

Rosson, S., Fuentealba, C., Hormazábal, C., Villena, C. y Brieba, F. (2013). Enfermedad de Parkinson y demencia, calidad de vida y sobrecarga del cuidador. Intervención multidisciplinaria en Atención Primaria. Revista Chilena Salud Pública, 17, 48-53.

Salgado, M. y Espinosa, G. (2012). Proceso de atención de enfermería a un paciente con Parkinson más psicosis basado en las 14 necesidades de Virginia Henderson. Enfermería Neurológica, 11, 81-86.

Scott, J. y Huskisson, E. C. (1979). Vertical or horizontal visual analogue scales. Annals of the Rheumatic Diseases, 38, 560.

Tinetti, M. E. (1986). Performance-oriented assessment of mobility problems in elderly patients. Journal of American Geriatric Society, 34, 119-126. 